ACCEPTED MANUSCRIPT

\title{
Measurement of mechanical losses in the carbon nanotube black coating of silicon wafers
}

To cite this article before publication: Leonid Prokhorov et al 2019 Class. Quantum Grav. in press https://doi.org/10.1088/1361-6382/ab5357

\section{Manuscript version: Accepted Manuscript}

Accepted Manuscript is "the version of the article accepted for publication including all changes made as a result of the peer review process, and which may also include the addition to the article by IOP Publishing of a header, an article ID, a cover sheet and/or an 'Accepted

Manuscript' watermark, but excluding any other editing, typesetting or other changes made by IOP Publishing and/or its licensors"

This Accepted Manuscript is @ 2019 IOP Publishing Ltd.

During the embargo period (the 12 month period from the publication of the Version of Record of this article), the Accepted Manuscript is fully protected by copyright and cannot be reused or reposted elsewhere.

As the Version of Record of this article is going to be / has been published on a subscription basis, this Accepted Manuscript is available for reuse under a CC BY-NC-ND 3.0 licence after the 12 month embargo period.

After the embargo period, everyone is permitted to use copy and redistribute this article for non-commercial purposes only, provided that they adhere to all the terms of the licence https://creativecommons.org/licences/by-nc-nd/3.0

Although reasonable endeavours have been taken to obtain all necessary permissions from third parties to include their copyrighted content within this article, their full citation and copyright line may not be present in this Accepted Manuscript version. Before using any content from this article, please refer to the Version of Record on IOPscience once published for full citation and copyright details, as permissions will likely be required. All third party content is fully copyright protected, unless specifically stated otherwise in the figure caption in the Version of Record.

View the article online for updates and enhancements. 


\title{
1. Introduction
}

\begin{abstract}
The successful detection of gravitational waves from astrophysical sources carried out by the laser interferometric detectors LIGO and Virgo have stimulated scientists to develop a new generation of more sensitive gravitational wave detectors. In the proposed upgrade called LIGO Voyager, silicon test masses will be cooled to cryogenic temperatures. To provide heat removal from the test masses when they absorb the laser light one can increase their thermal emissivity using a special black coating. We have studied mechanical losses in a carbon nanotube black coating deposited on silicon wafers. The addítional thermal noise associated with mechanical loss in this coating was calculated using a value of the product of the coating Young's modulus and the coating mechanical loss angle determined from the measurements. It was found that at temperatures of about $123 \mathrm{~K}$, the additional thermal noise of the LIGO Voyager test mass caused by the carbon nanotube black coating deposited on its barrel is less than the noise associated with the Acktar Black coating and is 20 times less than the noise due to the optical high reflective (HR) coating of the test mass.
\end{abstract}

The detections of gravitational waves by the laser interferometric detectors LIGO and Virgo have stimulated scientists to develop a new generation of gravitational wave detectors significantly superior in sensitivity [1,2]. Cooling the detector's test masses will reduce their thermal noise, including the thermal noise due to the test mass mirror coatings deposited. Thermal noise $[3,4]$ is one of the fundamental limits to the detector's sensitivity. The thermal noise power spectral density for mirrors is proportional to the mirror temperature and the mechanical loss angle. Unlike fused silica, in silicon the loss decreases when cooled below room temperature [5]. Silicon also has good thermal and optical properties at wavelengths of $1.5-2.5 \mu \mathrm{m}[6,7]$. In the LIGO Voyager project, the fused silica test-mass substrates and suspensions will be replaced with crystalline silicon and kept at $123 \mathrm{~K}$. It is likely that cooled silicon test masses will also be used 


\section{Measurement of mechanical losses in carbon nanotube black coating of silicon wafers 2}

in projects Cosmic Explorer and Einstein Telescope. The thermal noise associated with (Zener) thermoelastic damping is highly suppressed in silicon at $123 \mathrm{~K}$ due to the zerocrossing in its thermal expansion coefficient $[8,9]$. In order to maintain this operating temperature it will be necessary to extract up to $10 \mathrm{~W}$ of the heat absorbed by the test mass from the laser beam under high vacuum conditions [10]. This can be done by radiative cooling, but the thermal emissivity of the silicon test mass and the optical $\mathrm{HR} / \mathrm{AR}$ coatings is insufficient to provide the necessary rate of radiative cooling [11]. For example, the test mass barrels will need to be partially coated with high emissivity coatings such as Diamond-Like Carbon (DLC) [12], Acktar Black [13], or the carbon nanotube Advanced Nanophotonics black coating [14]. An important consideration is the additional thermal noise associated with the mechanical loss in this coating. The mechanical loss of the Acktar Black coating was explored in [15], and it was shown that the Acktar coating results in a $\sim 9 \%$ increase of the total strain noise of LIGO Voyager. It is therefore interesting and important to explore other high-emissivity coatings that produce lower thermal noise. In this paper, we present results of an investigation into the mechanical loss in the temperature range of $100-295 \mathrm{~K}$ of the carbon nanotube Advanced Nanophotonics black coating deposited on silicon wafers.

\section{Experimental setup}

The carbon nanotube Advanced Nanophotonics black coating is made of a thin layer of vertically aligned multi-walled carbon nanotubes. The nanotubes were grown on a silicon wafer using a catalyst assisted chemical vapor deposition (CVD) technique [16]. The coating absorbs about $99 \%$ of the incident radiation, when measured at roomtemperature and at $5 \mathrm{~K}$ over a broad wavelength range of $0.4-200 \mu \mathrm{m}$ [17]. The carbon nanotube black coating was deposited on one face of a commercial (Virginia Semiconductor Inc.) double side polished silicon $<100>$ wafer with diameter of $51 \mathrm{~mm}$ and thickness of $0.27 \mathrm{~mm}$. The wafers have two flats as shown in Figure 1. The flats with length of $16 \mathrm{~mm}$ and $8 \mathrm{~mm}$ cut into opposite sides of the wafer indicate the crystallographic planes and that the wafer is fabricated from n-type silicon. We investigated three types of carbon nanotube Advanced Nanophotonics black coatings, differing in the buffer layer material, iron catalyst layer thickness, and in coating deposition technology [16]. The coating layer thickness is $t_{c}=(100 \pm 50)$ micron and the coating material density $\rho_{c} \approx 30 \mathrm{~kg} / \mathrm{m}^{3}$.

We used the coated silicon wafer as a disk-shaped mechanical resonator. Such resonators are widely used in order to measure mechanical loss angle in thin film coatings deposited on the resonator's surface [18, 19]. A gentle nodal suspension (GeNS) of disk resonators allows a significant reduction of losses coming from the suspension system [20, 21]. We use a nodal suspension of the wafer with clamping between two stems instead of a GeNS because it is more robust to hold the wafer during transfer into the cryogenic vacuum chamber and it also provides negligible level of clamping loss. Ideally, one would measure the mechanical loss for the uncoated wafers, coat 
Measurement of mechanical losses in carbon nanotube black coating of silicon wafèrs 3

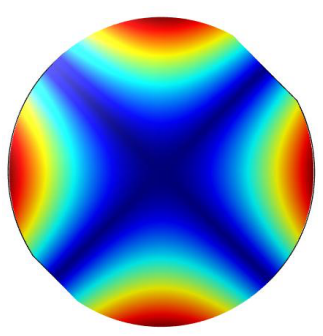

a) $f=824 \mathrm{~Hz}$

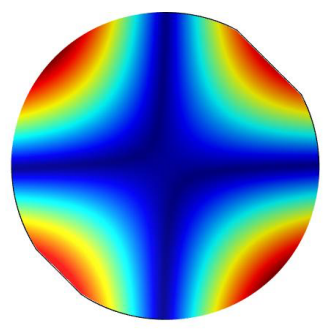

b) $f=1011 \mathrm{~Hz}$

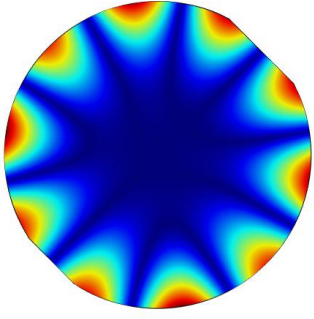

c) $f=5611 \mathrm{~Hz}$

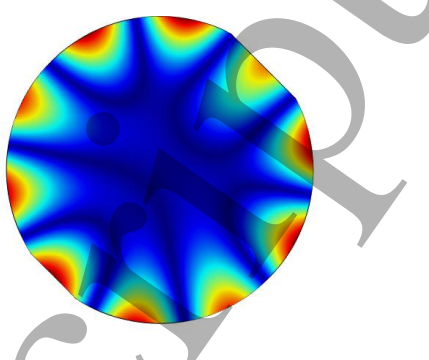

d) $f=5627 \mathrm{~Hz}$

Figure 1. Calculated distribution of the total displacement for vibration modes with two $(\mathrm{a}, \mathrm{b})$ and five $(\mathrm{c}, \mathrm{d})$ nodal diameters. The wafer has two flats cut into opposite sides for indication of crystallographic planes and silicon doping type.

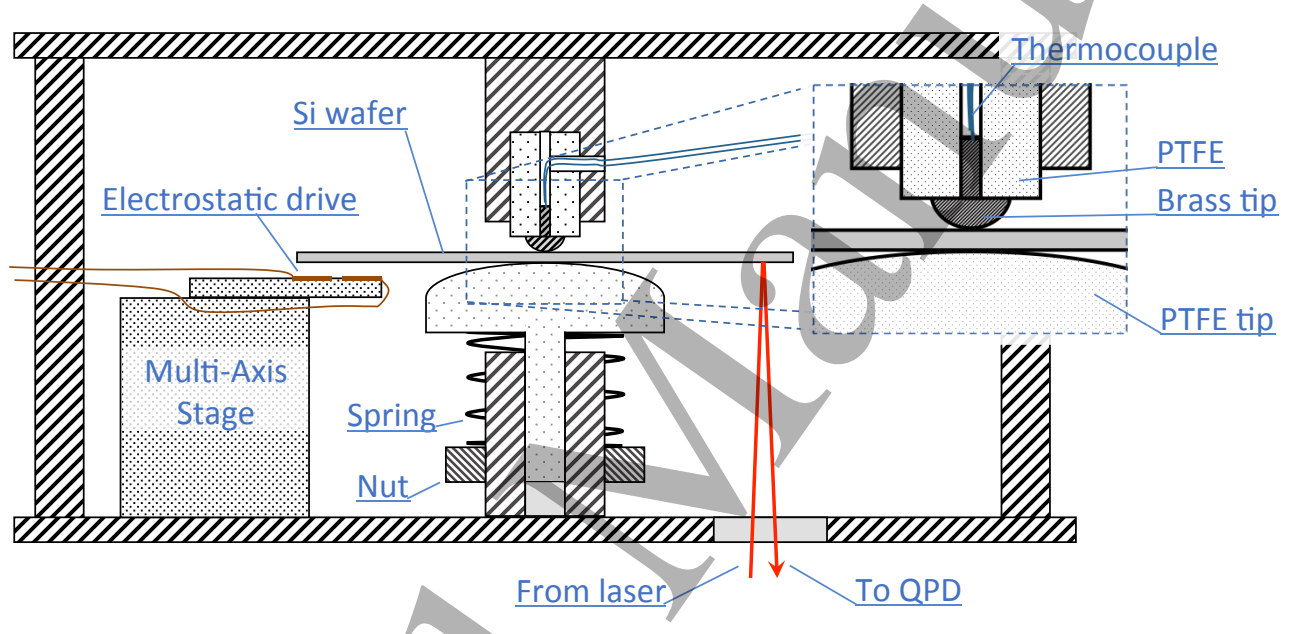

Figure 2. Schematic side view of the frame with clamped silicon wafer.

them, and then measure the coated wafer mechanical loss. Since this would increase the overall experiment time, we instead measured a number of uncoated reference wafers as a baseline and compared the results with the coated disks. This method somewhat increases the uncertainty in the inferred value of the product of the coating Young's modulus and the coating mechanical loss angle determined from the measurements, but this uncertainty makes only a small contribution to the total uncertainty of the value of the product.

The silicon wafer is centrally clamped between two stems attached to an aluminum frame as is shown in Figure 2. The bottom stem had a PTFE (Teflon) tip of a hemispherical shape, the brass tip of a hemispherical shape is mounted on the upper stem through an intermediate PTFE gasket. The gasket slows the process of heat transfer from the wafer to the cold stem. The bottom stem is mounted in a springloaded manner, which stabilizes the clamping force when the temperature changes.

The frame with the clamped wafer is attached to a dewar with liquid nitrogen mounted inside the vacuum chamber. The wafer vibration is excited at the resonant frequency of a mode using an electrostatic drive. The wafer vibration amplitude is monitored by an optical sensor; the local bending of the wafer produced by its vibration 


\section{Measurement of mechanical losses in carbon nanotube black coating of silicon wafers 4}

results in a deflection of the laser beam reflected from the peripheral region of the wafer surface. The distance between the laser spot and the edge of the wafer is $3-5 \mathrm{~mm}$. The reflected beam passes through a system of mirrors to steer the light and is detected by a split quadrant photodiode (QPD) placed outside the vacuum chamber. The mechanical loss equal to the reciprocal of the mechanical quality factor $Q^{-1}$ is determined from the ring-down time $\tau$ of a resonant mode according to the relation $Q=\pi f \tau$, where $f$ is the resonant mode frequency. The temperature of the wafer is measured using a thermocouple sensor attached to the brass tip which was in direct thermal contact with the wafer. Measurements of the temperature dependent mechanical loss were made during the wafer cooling after the dewar was filled with liquid nitrogen. In the most interesting temperature range of $100-150 \mathrm{~K}$, the results coincided with those measured during the wafer heating after evaporation of liquid nitrogen, which indicates a good thermal contact between the thermocouple sensor and the wafer. Measurements are carried out in vacuum under a residual pressure of about $10^{-6}$ Torr. The residual gas damping gives a negligible contribution to the measured mechanical loss. A more detailed description of the experimental setup and the measurement procedure can be found in [15].

\section{Results of measurements and discussion}

We measured the losses of 5 eigen-modes of the silicon wafers. The selected modes have a number of nodal diameters from 2 to 6 and zero nodal circles. The distributions of the total displacement for vibration modes with two and five nodal diameters calculated using a finite element method (FEM) are shown in Figure 1. The vibrations are concentrated in the circumferential region of the wafer. The clamping losses are small for these modes. Modes are identified by comparing their measured resonant frequencies with those calculated by FEM. In these calculations, the anisotropic elasticity matrix of silicon with elastic constants $C_{11}=166 \mathrm{GPa}, C_{12}=64 \mathrm{GPa}$ and $C_{44}=80 \mathrm{GPa}$ was used [22]. Each mode has two split frequencies due to the anisotropy of silicon and the flats cut into the wafers. The splitting of frequencies is small in comparison with the frequencies themselves except for a mode with two nodal diameters. The mode with two nodal diameters has two split frequencies at 833 and $1029 \mathrm{~Hz}$. The approximate resonant frequencies of the other modes are $2.1,3.6,5.6$, and $7.8 \mathrm{kHz}$. The mechanical loss of the split frequencies of these modes differ by tens of percent, apparently due to the different clamping loss. For each mode, the one of the two split frequencies which has the lowest mechanical loss is considered. The mechanical loss angle of the carbon nanotube coating. $\phi_{c}$, can be written as a function of the difference between the measured mechanical loss of the bending modes of the coated and uncoated wafers [15, 23]:

$$
\phi_{c}=k \frac{Y_{s} t_{s}}{Y_{c} t_{c}}\left(Q_{\text {coated }}^{-1}-Q_{\text {uncoated }}^{-1}\right)
$$

Equation (1) is obtained using the assumption that the substrate and the coating are isotropic materials, where $Y_{s}$ and $t_{s}$ are the Young's modulus and thickness of the 
Figure 3. Relative difference in mode frequencies measured for 5 silicon wafers (\#1, 2 - uncoated wafers, \#3, 4, 5 - wafers with coating, wafer \#2 is used as a reference).

substrate material, respectively, $Y_{c}$ and $t_{c}$ are the Young's modulus and thickness of the coating. The coefficient, $k$, is obtained from the FEM, assuming that the coating layer is described by equations of solid mechanics. For the thin coating layer, when $t_{c}<<t_{s}$, $k \approx 0.3[15,23]$. The coefficient $k$ depends weakly on the value of the Poisson ratios $\nu_{s}$ and $\nu_{c}$. If the coating layer is not thin compared to the thickness of the silicon wafer, $k$ depends on the Young's modulus of the substrate and the coating materials. We have no information about the Young's modulus of the coating material. The manufacturer gives the thickness of the coating layer, and its density. The Young's modulus of the coating material (considered as an isotropic material) can be estimated from the change in the mode frequency after coating of the wafer, as shown in the next paragraphs.

The relative difference in the $5.6 \mathrm{kHz}$ mode frequencies measured for the 5 silicon wafers (3 coated and 2 uncoated) that we had at our disposal is shown in Figure 3 (diamonds). The uncoated wafer \#2 is used as a reference. The measurement error of the relative frequency difference does not exceed $3 \%$. A similar situation was observed for the other modes. The maximal data spread was obtained for the $0.8 \mathrm{kHz}$ mode which is denoted by squares. One can see that the measured relative difference in mode frequencies does not exceed $2 \%$. If the frequency difference were determined only by the coating, then it would vary depending on the Young's modulus of the coating as shown in Figure 4. Notice that there is a contribution to the difference of frequencies which we cannot control. It is caused by variations of the uncoated silicon wafer thickness used for deposition of the coating. Despite a small amount of data, we can conclude that we do not detect a noticeable change in the frequency of the mode for coated wafers, i.e. the coating Young's modulus does not exceed $5 \mathrm{GPa}$. This conclusion agree with the data obtained in the work [24].

Denote the ratio $\frac{Q_{\text {coated }}^{-1}-Q_{\text {uncoated }}^{-1}}{Y_{c} \phi_{c}}$ which is included in Equation (1) by the letter $D$. The value of $D$ for the $5.6 \mathrm{kHz}$ mode as a function of $Y_{c}$, in the range of $1-10 \mathrm{GPa}$, 
Measurement of mechanical losses in carbon nanotube black coating of silicon wafèrs 6

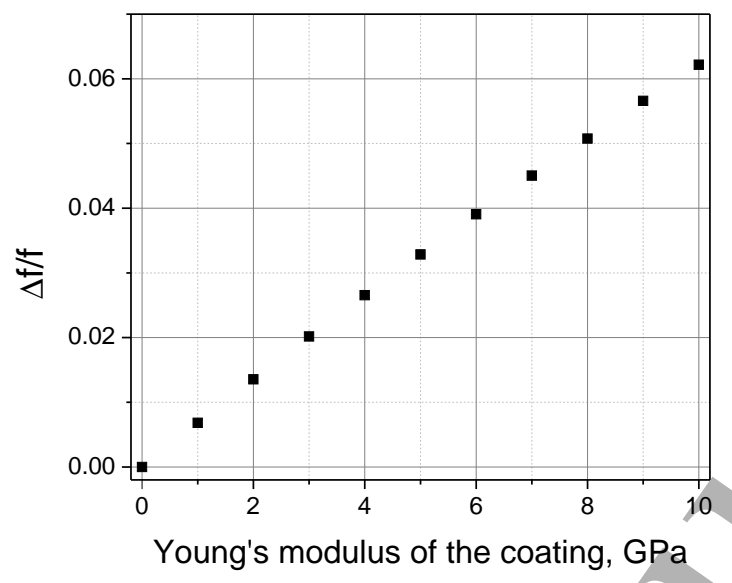

Figure 4. Relative change of the $5.6 \mathrm{kHz}$ mode frequency as a function of the coating Young's modulus computed by FEM. The elastic properties of silicon are described by the elasticity matrix with constants $C_{11}=166 \mathrm{GPa}, C_{12}=64 \mathrm{GPa}$ and $C_{44}=80 \mathrm{GPa}$. Non-variable parameters are the following: $t_{s}=0.27 \mathrm{~mm}, t_{c}=0.1 \mathrm{~mm}, \nu_{c}=0.27$, $\rho_{s}=2300 \mathrm{~kg} / \mathrm{m}^{3}, \rho_{c}=30 \mathrm{~kg} / \mathrm{m}^{3}$.

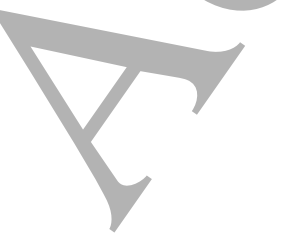

Figure 5. The ratio $D=\frac{Q_{\text {coated }}^{-1}-Q_{\text {uncoated }}^{-1}}{Y_{c} \phi_{c}}$ for $5.6 \mathrm{kHz}$ mode as a function of the coating Young's modulus computed by FEM. Elastic properties of silicon are described by the elastic matrix with constants $C_{11}=166 \mathrm{GPa}, C_{12}=64 \mathrm{GPa}$ and $C_{44}=80 \mathrm{GPa}$. Non-variable parameters are the following: $t_{s}=0.27 \mathrm{~mm}, t_{c}=0.1 \mathrm{~mm}, \nu_{c}=0.27$, $\rho_{s}=2300 \mathrm{~kg} / \mathrm{m}^{3}, \rho_{c}=30 \mathrm{~kg} / \mathrm{m}^{3}$. 


\section{Measurement of mechanical losses in carbon nanotube black coating of silicon wafers 7}

computed by FEM, is shown in Figure 5 . It can be concluded that $\left(Q_{\text {coated }}^{-1}-Q_{\text {uncoated }}^{-1}\right)$ is approximately proportional to $Y_{c}$. The change in the coefficient of proportionality does not exceed $10 \%$ in the range of $0-5 \mathrm{GPa}$. Substituting the values of parameters in Equation (1), we obtain the value of the coefficient $k$. Since the formula was obtained for an isotropic model, the value of the isotropic Young's modulus and Poisson ratio of silicon $Y_{s}=156 \mathrm{GPa}, \nu_{s}=0.27$ were used. We can also use $k=0.18 \pm 0.01$. We cannot determine the coating mechanical loss angle from this formula because we do not know the coating $Y_{c}$ precisely. We can, however, place bounds on it for estimation purposes. We can find a value of the product $Y_{c} \phi_{c}$ from the measured difference $\left(Q_{\text {coated }}^{-1}-Q_{\text {uncoated }}^{-1}\right)$. FEM computation of the test mass thermal noise caused by the barrel coating shows that the displacement noise power spectral density is proportional to this product rather than $Y_{c}$ and $\phi_{c}$, individually.

The temperature dependences of the loss of the first five bending modes measured in the uncoated silicon wafer are shown in Figure 6. The thermoelastic losses were calculated for the $0.8 \mathrm{kHz}$ and $7.6 \mathrm{kHz}$ modes using the COMSOL Multiphysics FEM package [25]. They are shown with dashed and solid lines. The temperature dependent thermodynamic parameters of silicon were taken from [26, 27]. For high-frequency modes the measured losses are close to the calculated thermoelastic loss everywhere except in the temperature range near $123 \mathrm{~K}$, where they are suppressed due to the approach to zero of the coefficient of thermal expansion of silicon. For the two low-frequency modes, higher levels of mechanical losses are observed as well as a broad loss peak at around $180 \mathrm{~K}$. In common with [28], we believe these are not intrinsic to the silicon material but are likely contaminated by clamping losses.

The temperature dependences of miechanical loss of the same five bending modes measured in the wafer with carbon nanotube black coating which was deposited on the wafer using an alumina buffer layer and water-assisted growth technique [15] are shown in Figure 7. For this type of carbon nanotube black coating of the three measured types the least mechanical loss was obtained. The most interesting temperature range is near $123 \mathrm{~K}$. The difference between losses of coated and uncoated wafers measured for different modes at $123 \mathrm{~K}$ is shown in Figure 8. The error bars of these values are determined by uncertainties of the measured mechanical loss, and are likely associated with clamping of the wafer. In order to reduce errors associated with clamping loss the wafer was re-clamped at least three times and mechanical loss measurements repeated. We cannot conclude anything definitive about the measured frequency dependence of the coating mechanical loss, and we therefore assume that the mechanical loss in the coating is requency independent in the range $(0.8-8 \mathrm{kHz})$.

The measured mechanical loss includes the mechanical loss (internal friction) and the thermoelastic loss arising due to non-zero coefficient of the thermal expansion $[29,30]$. The thermoelastic loss depends not only on thermodynamic and elastic properties of the material but also on the kind of deformations and their distribution which may differ in the case of different modes of a coated thin disc and a cylinder with a coated barrel. Therefore, the thermoelastic loss and noise should be calculated 


\section{Measurement of mechanical losses in carbon nanotube black coating of silicon wafèrs 8}

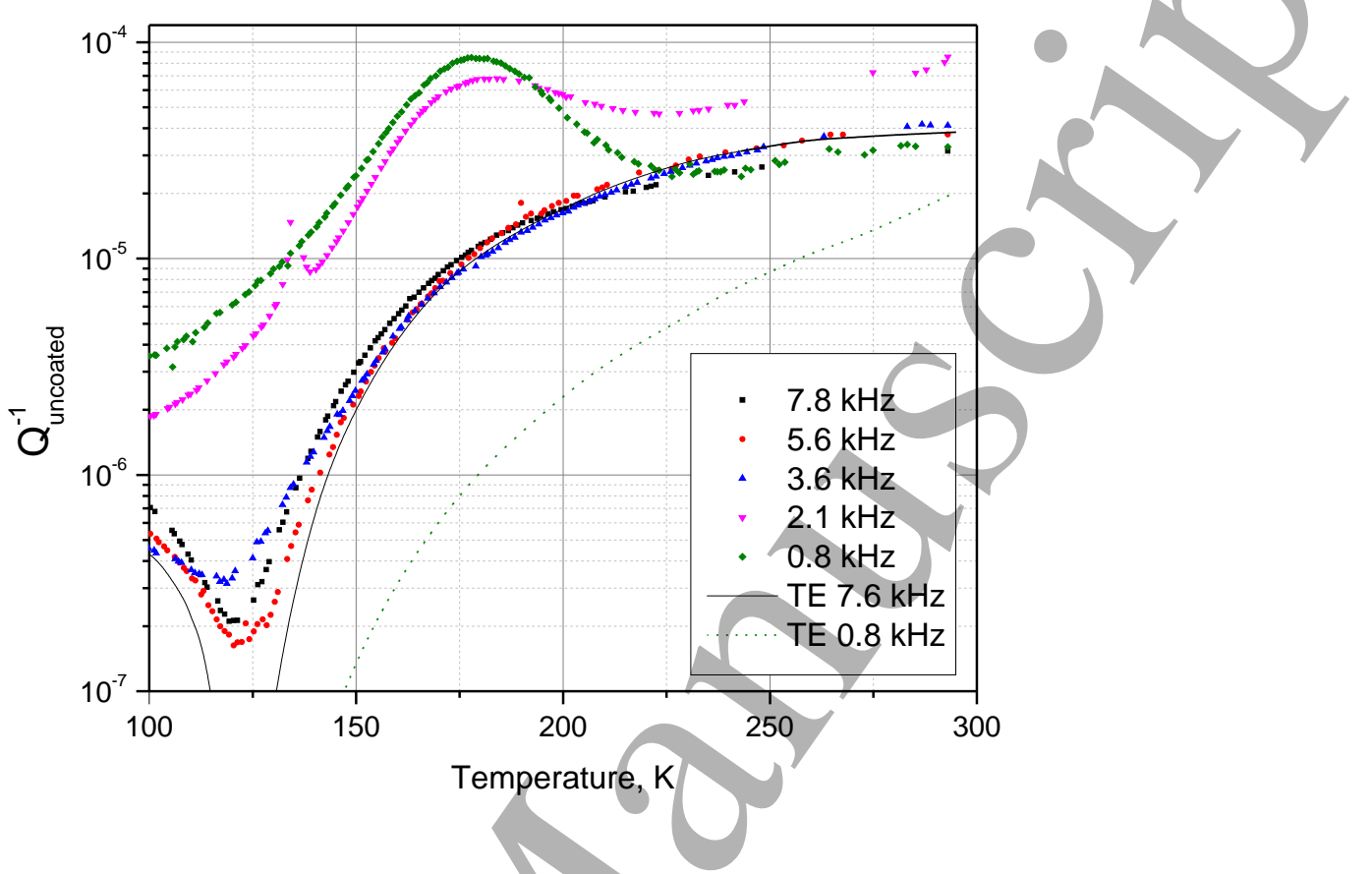

Figure 6. Temperature dependence of the mechanical loss of different modes in an uncoated silicon wafer. The thermoelastic losses calculated for the $0.8 \mathrm{kHz}$ and $7.6 \mathrm{kHz}$ modes are shown with dashed and solid lines.

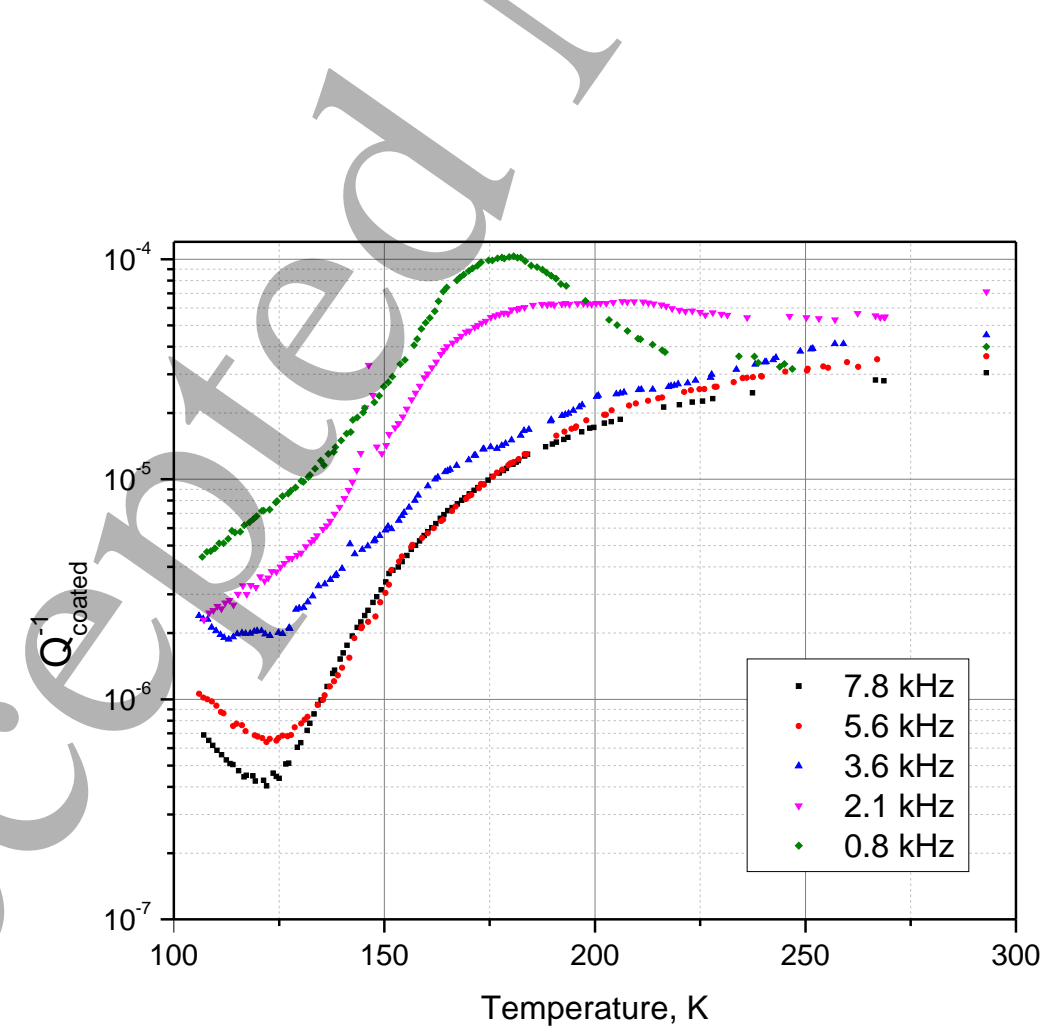

Figure 7. Temperature dependence of mechanical loss of different modes in a coated silicon wafer. 
1

2

3

4

5

6

7

8

\section{Measurement of mechanical losses in carbon nanotube black coating of silicon wafèrs 9}

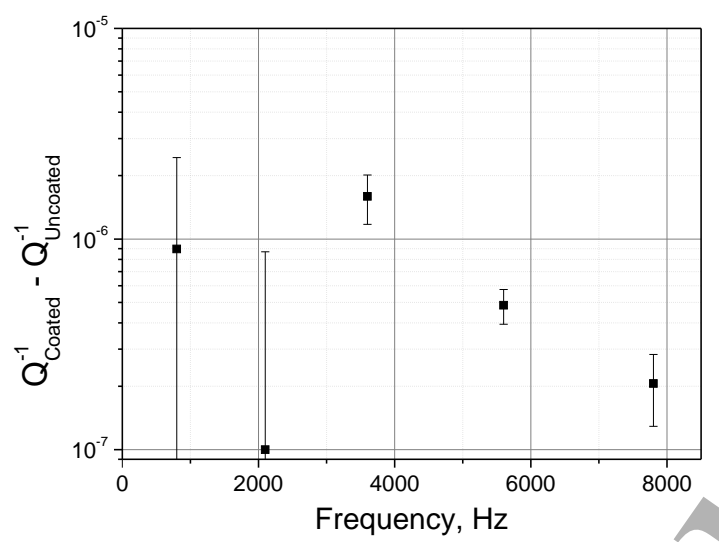

Figure 8. The difference between losses of coated and uncoated wafers measured for different modes at temperature near $123 \mathrm{~K}$.

separately from those determined by the mechanical loss. In order to calculate thermoelastic loss and noise of the coating we need to know the thermodynamic properties of the black coating material. We have not found these parameters in the literature. Since we cannot separate the thermoelastic loss of the coating we consider the measured mechanical loss as the upper limit of the mechanical loss of the coating. Then the average value of the difference between losses of coated and uncoated silicon wafers measured for different modes in this/frequency range, at the temperature of minimal loss near $123 \mathrm{~K}$, is $(4 \pm 1) \times 10^{-\pi}$. The magnitude of the product of $Y_{c} \phi_{c}$ is defined from Equation (1). Its uncertainty is determined by uncertainties of the parameters in this formula. The main contribution to the uncertainty is given by errors of $\left(Q_{\text {coated }}^{-1}-Q_{\text {uncoated }}^{-1}\right), t_{c}$ and $k$. As a result, we obtain $Y_{c} \phi_{c}=(3.0 \pm 1.7) \times 10^{4} \mathrm{~Pa}$. This value is much smaller than the analogous value of $Y_{c} \phi_{c}=(3.1 \pm 0.7) \times 10^{7} \mathrm{~Pa}$ obtained for the Acktar Black coating [15].

Notice that this value is obtained for the specific coating which we have investigated. Changes in the technology of its deposition including the coating layer thickness can result in a change of this value. The obtained value of the product $Y_{c} \phi_{c}$ was used for FEM calculation of the LIGO Voyager test mass displacement thermal noise associated with the carbon nanotube black coating of the test mass barrel. It is shown by light blue line in Figure 9. All other noise sources are from the baseline LIGO Voyager design $[7,31]$. The calculation details can be found in [15]. It can be seen that the noise caused by the carbon nanotube black coating makes an insignificant contribution to the total noise of the test masses.

\section{Conclusion}

In future cryogenic interferometric gravitational wave detectors, heat removal from silicon test masses could be be assisted if their barrel surfaces are coated with the carbon 
Measurement of mechanical losses in carbon nanotube black coating of silicon wafèrs 10

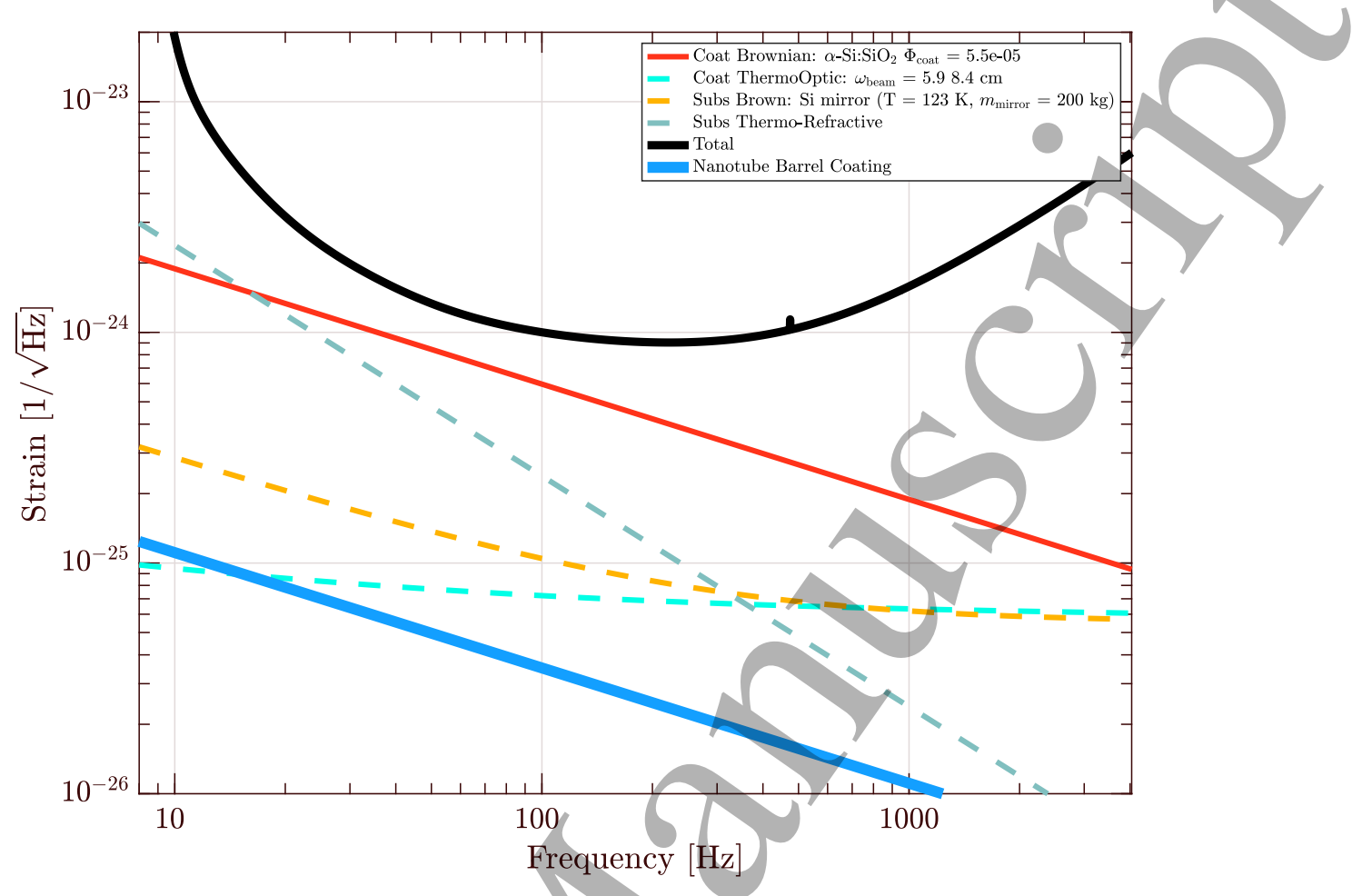

Figure 9. Strain sensitivity of LIGO Voyager. Total noise is shown by black line. The estimated Brownian thermal noise contribution from the carbon nanotube barrel coating is shown by bold blue line. Noise sources are from the baseline LIGO Voyager design: $200 \mathrm{~kg}$ silicon test mass at $123 \mathrm{~K}, 3 \mathrm{MW}$ of circulating laser power with a 2-micron wavelength. The contributions of other noise sources are also shown for comparison: Brownian thermal noise from the mirror HR surface coating of amorphous silicon and silica (red line), this coating thermo-optic noise (dashed cyan line), substrate Brownian thermal noise (dashed orange line), substrate thermorefractive noise (dashed grey line) [7, 31].

nanotube Advanced Nanophotonics black coating. In order to calculate additional test mass displacement thermal noise associated with mechanical loss of this coating, we used a value of product of the coating Young's modulus and the coating mechanical loss angle rather than these parameters of the coating determined individually. We have determined this value for the carbon nanotube Advanced Nanophotonics black coating using measurements of the mechanical loss of bending vibration modes of coated and uncoated commercial silicon wafers. It was found that the additional thermal noise of the test mass displacement caused by the carbon nanotube black coating deposited on its barrel is less than the noise associated with the Acktar Black coating and is 20 times less than the noise due to the optical HR test mass coating. In addition to high emissivity and low mechanical loss, the ability to clean is an important requirement for the coating deposited on the test mass. Further research is needed to find the optimal cleaning procedure for the carbon nanotube black coating, which would retain its high emissivity and low mechanical loss. 


\section{Measurement of mechanical losses in carbon nanotube black coating of silicon wafers 11}

\section{Acknowledgments}

The authors gratefully acknowledge the support of the Russian Science Foundation Grant No. 17-12-01095 and the MSU Fund at TAPIR. LIGO was constructed by the California Institute of Technology and Massachusetts Institute of Technology with funding from the National Science Foundation and operates under cooperative agreement PHY-0757058. This paper has LIGO Document Number LIGO-P1800369.

\section{References}

[1] B. P. Abbott, R. Abbott, T. D. Abbott, et al. Observation of gravitational waves from a binary black hole merger. Phys. Rev. Lett., 116:061102, 2016.

[2] B. P. Abbott, R. Abbott, T. D. Abbott, et al. GW170817: Observation of gravitational waves from a binary neutron star inspiral. Phys. Rev. Lett., 119:161101, 2017.

[3] Y. Levin. Internal thermal noise in the LIGO test masses: A direct approach. Phys. Rev. D, 57:659-663, 1998.

[4] Stuart Reid and Iain W. Martin. Development of mirror coatings for gravitational wave detectors. Coatings, 6(4):61, 2016.

[5] Ronny Nawrodt, Sheila Rowan, Jim Hough, Michele Punturo, Fulvio Ricci, and Jean-Yves Vinet. Challenges in thermal noise for 3rd generation of gravitational wave detectors. General Relativity and Gravitation, 43(2):593-622, Feb 2011.

[6] S. Rowan, R. L. Byer, M. M. Fejer, et al. Test mass materials for a new generation of gravitational wave detectors. Proc. SPIE, 4856:292-297, 2003.

[7] R. X. Adhikari. Gravitational radiation detection with laser interferometry. Rev. Mod. Phys., 86:121-151, 2014.

[8] C. A. Swenson. Recommended values for the thermal expansivity of silicon from 0 to $1000 \mathrm{~K}$. J. Phys. Chem. Ref. Data, 12(2):179-182, 1983.

[9] D. S. Kim, O. Hellman, J. Herriman, et al. Nuclear quantum effect with pure anharmonicity and the anomalous thermal expansion of silicon. Proc. NAS, 115(9):1992-1997, 2018.

[10] R. Adhikari, N. Smith, A. Brooks, et al. LIGO Voyager upgrade conceptual design. LIGO Technical Note, T1400226-v7, 2016.

[11] R. Weiss. Silicon mirrôr cooled to 120 K. LIGO Technical Note, T1200093, 2012.

[12] Y. Sakakibara, N. Kimura, T. Suzuki, et al. A study of cooling time reduction of interferometric cryogenic gravitational wave detectors using a high-emissivity coating. AIP Conf. Proc., 1573(1):1176-1183, 2014.

[13] Acktar Ltd. Black coating services. http://www.acktar.com/category/BlackOpticalCoating.

[14] John Hagopian, Manuel Quijada, June Tveekrem, et al. Multiwalled carbon nanotubes for stray light suppression in space flight instruments. Proc. of SPIE, 7761, 2010.

[15] M R Abernathy, N Smith, W Z Korth, R X Adhikari, L G Prokhorov, D V Koptsov, and V P Mitrofanov. Measurement of mechanical loss in the Acktar Black coating of silicon wafers. Class. Quant. Grav., 33(18):185002, 2016.

[16] J. Lehman, C. Yung, N. Tomlin, et al. Carbon nanotube-based black coatings. Appl. Phys. Rev., 5(1):011103, 2018.

[17] John Lehman, Andreas Steiger, Nathan Tomlin, Malcolm White, Mathias Kehrt, Ivan Ryger, Michelle Stephens, Christian Monte, Ingmar Mueller, Joerg Hollandt, and Marla Dowell. Planar hyperblack absolute radiometer. Opt. Express, 24(23):25911-25921, Nov 2016.

[18] M. Granata, L. Balzarini, J. Degallaix, et al. Internal friction and Young's modulus measurements on $\mathrm{SiO} 2$ and Ta2O5 films done with an ultra-high Q silicon-wafer suspension. Arch. Metall. and Mater., 60(1):365-370, 2015. 
Measurement of mechanical losses in carbon nanotube black coating of silicon wafers 12

[19] G. Cagnoli, M. Lorenzini, E. Cesarini, et al. Mode-dependent mechanical losses in disc resonators. Phys. Lett. A, 382(33):2165 - 2173, 2018. Special Issue in memory of Professor V.B. Braginsky.

[20] E. Cesarini, M. Lorenzini, E. Campagna, et al. A "gentle" nodal suspension for measurements of the acoustic attenuation in materials. Rev. Sci. Instrum., 80(5):053904, 2009.

[21] G. Vajente, A. Ananyeva, G. Billingsley, et al. A high throughput instrument to measure mechanical losses in thin film coatings. Rev. Sci. Instrum., 88(7):073901, 2017.

[22] M. A. Hopcroft, W. D. Nix, and T. W. Kenny. What is the Young's modulus of silicon? J. Microelectromech. Syst., 19(2):229-238, 2010.

[23] K. Yamamoto, S. Miyoki, T. Uchiyama, et al. Measurement of the mechanical loss of a cooled reflective coating for gravitational wave detection. Phys. Rev. D, 74:022002, 2006.

[24] John L. Rigueur, Saad A. Hasan, Sameer V. Mahajan, and James H. Dickerson. Buckypaper fabrication by liberation of electrophoretically deposited carbon nanotubes. Carbon, 48(14):4090 $-4099,2010$.

[25] Comsol multiphysics: the platform for physics-based modeling and simulation. https://comsol.com/comsol-multiphysics.

[26] P. D. Desai. Thermodynamic properties of iron and silicon. Journal of Physical and Chemical Reference Data, 15(3):967-983, 1986.

[27] C. Y. Ho, R. W. Powell, and P. E. Liley. Thermal conductivity of the elements. Journal of Physical and Chemical Reference Data, 1(2):279-421, 1972.

[28] S. Reid, G. Cagnoli, D.R.M. Crooks, et al. Mechanical dissipation in silicon flexures. Phys. Lett. A, 351:205-211, 2006.

[29] Clarence Zener. Internal friction in solids. I. Theory of internal friction in reeds. Phys. Rev., 52:230-235, 1937.

[30] Ron Lifshitz and Michael Roukes. Thermoelastic damping in micro- and nanomechanical systems. Phys. Rev. B, 61:5600-5609, 2000.

[31] Instrument science white paper 2018. LIGO Technical Note, T1800133, 2018. 